Revista Perspectivas Online: Exatas \& Engenharias Dezembro de 2017, Vol.7, no 19, p. 52-62

ISSN: $2236-885 \mathrm{X}$ (Online)

DOI: $10.25242 / 885 \times 7192017958$

\title{
ANÁLISE DE CAPACIDADE DO PROCESSO DE PESAGEM DE LINGÜIÇA TOSCANA EM UMA INDÚSTRIA ALIMENTÍCIA
}

\author{
Thiago Correa Leite ${ }^{1 *}$, Cidmar Ortiz dos Santos' ${ }^{1}$, José Airton Azevedo dos Santos ${ }^{1}$ e Carla \\ Adriana Pizarro Schmidt ${ }^{1}$
}

\section{RESUMO}

LEITE, T.C.; SANTOS, C.O., SANTOS, J.A.A.; SCHIMIDT, C.A.P. Analise de capacidade do processo de pesagem de linguica toscana em uma industria alimenticia. Perspectivas Online: Exatas \& Engenharias, v. 7 , n. 19, p. 52-62,2017.

Para a realização do controle estatístico do processo são utilizadas várias ferramentas, dentre elas a análise da capacidade. Este é um método que tem o objetivo de verificar se um processo é considerado estatisticamente capaz de atender as especificações do cliente e se está atuando dentro das especificações ideais projetadas. Diante desta premissa, o estudo realizado em uma indústria alimentícia, teve como foco analisar a estabilidade e a capacidade de um processo de produção de linguiça toscana por meio de gráficos de controle e cálculo da capacidade do processo. Para tanto, foram pesadas sessenta e seis embalagens de $1 \mathrm{~kg}$ de lingüiça toscana. Posteriormente, verificou-se a estabilidade, a normalidade dos dados e o índice de capacidade $\mathrm{C}_{\mathrm{pk}}$. Observou-se que o processo apresentava causas especiais e que o índice de capacidade $\mathrm{C}_{\mathrm{pk}}$, está acima de um, o que classifica o processo como razoavelmente capaz.

Palavras-chave: Controle Estatístico de Processo; Capacidade; Indústria Alimentícia. 


\begin{abstract}
To perform the statistical process control are used several tools, among them the capacity analysis. This is a method that aims to verify whether a process is considered statistically able to meet customer specifications and is acting within the ideal specifications designed. Given this premise, the study carried out in a food industry, focused on analyzing the stability and capacity of a process of

control charts and calculation of the process capacity. Sixty-six packs of $1 \mathrm{~kg}$ of Tuscan sausage were weighed. Subsequently, the stability, the normality of the data and the capacity index $\mathrm{C}_{\mathrm{pk}}$ were verified. It was observed that the process had special causes and that the capacity index $\mathrm{C}_{\mathrm{pk}}$, is above one, which classifies the process as reasonably capable.
\end{abstract} production of Tuscan sausage by means of

Keywords: Statistical Process Control; Capacity; Food industry.

\footnotetext{
${ }^{1}$ Universidade Tecnológica Federal do Paraná (Campus Medianeira) - Coordenacao de Engenharia de Produção Avenida Brasil, 4236, Medianeira, Paraná, PR, CEP: 85884-000, Brasil;

(*)e-mail: leitetc@gmail.com

Data de chegada: 24/06/2016 Aceito para publicação: 14/11/2017
} 


\section{INTRODUÇÃO}

Em busca de conservar a carne o homen vem fabricando, desde a antiguidade, diferentes tipos de linguiça. Desde 1500 a. C. já é registrado pela história o consumo de linguiça pelos chineses e babilônios. Uma grande variedade de linguiças passou a ser comercializada, a partir da idade média, de acordo com o clima da região. Climas mais frios intesificaram as variedades frescais cruas ou defumadas, enquanto climas mais quentes enfatizaram embutidos desitradados (TERRA, 2000).

As linguiças, geralmente, variam em dois aspectos: o processo de preparação e o tipo de carne utilizado. A carne, em conjunto com os temperos, definem a maior parte do sabor. A linguiça pura utiliza somente a carne de um único animal, enquanto as mistas utilizam normalmente a carne de gado e de porco.

Em relação a preparação pode ser: fresca, curada ou defumada. As frescas precisam ser cozidas antes do consumo. As curadas são banhadas em uma mistura de sal, açucar e salitre. As defumadas passam por um processo de cozimento por fumaça, ou defumação (CLUBEDACARNE, 2017).

A linguiça toscana, produto de grande aceitação pelos brasileiros, é um produto cárneo de fácil preparo e com baixo custo de produção. Elaborada com matérias-primas provenientes do aproveitamento de cortes menos nobres de suínos e embutida em envoltório natural (TERRA, 2000).

Nos últimos anos observa-se que os consumidores apresentam um perfil bem mais seletivo e exigente, gerando assim uma maior competitividade entre as empresas alimentícias, levando-as a procurar vantagens competitivas. Segundo Juran (1993), algo fundamental para obtenção dessas vantagens competitivas é a melhoria dos processos produtivos. Esse tipo de melhoria pode ser alcançado de forma efetiva mediante a utilização de métodos estatísticos e ferramentas da qualidade. Tais métodos e ferramentas permitem que os dados gerados pelo processo possam ser interpretados, analisados e as conclusões tiradas (RAMOS, 2013).

As ferramentas principais utilizadas no controle estatístico de processos, cartas ou gráficos de controle, têm como objetivo detectar desvios de parâmetros representativos do processo, reduzindo a quantidade de produtos fora de especificações e os custos de produção (OLIVEIRA; LIMA, 2011).

Quando se verifica que o processo encontra-se sob controle estatístico, pode-se medir quanto esse processo consegue gerar produtos que atendam às especificações de projeto que refletem os desejos e exigências de seus clientes. Para isso, faz-se uso dos índices de capacidade do processo. Esses índices procuram detectar dois tipos de problemas. O primeiro é o de localização do processo, se o processo atende em média ao valor nominal de especificação, e o segundo, de variabilidade, quando o processo apresenta muita dispersão e não atende às especificações que são estabelecidas no projeto (OLIVEIRA et al., 2013).

Nesse contexto, este trabalho teve como objetivo analisar a estabilidade e a capacidade de um processo de produção de linguiça toscana por meio de gráficos de controle e cálculo da capacidade do processo.

O artigo é organizado da seguinte maneira. Na Seção 2 são descritas as metodologias empregadas na análise da variabilidade do peso das embalagens e da capacidade do processo (Controle Estatístico de Processo). Na Seção 3 são apresentados os resultados obtidos quando da aplicação da metodologia em questão a um conjunto de dados reais (pesos das embalagens de $1 \mathrm{~kg}$ de lingüiça toscana), juntamente com os resultados obtidos no software Statistica 11.0. Comentários finais e conclusões, na Seção 4, finalizam o artigo. 


\section{METODOLOGIA}

Tipo de Pesquisa:

A metodologia utilizada neste estudo pode ser classificada quanto ao tipo de pesquisa, objetivos e a forma de abordar o problema (GIL, 2002). A classificação quanto ao tipo de pesquisa pode ser subdividida de acordo com a natureza, sendo para este estudo aplicada, pois seus resultados são aplicados à resolução de problemas reais enfrentados pela empresa em estudo. Quanto aos objetivos pode ser classificada como descritiva, pois tem como objetivo descrever as características de determinada população ou fenômeno. Quanto à forma de abordar o problema pode ser considerada quantitativa, pois os dados obtidos no sistema real foram, em seguida, tratados estatisticamente.

Como estratégia de pesquisa, utilizou-se o estudo de caso que, conforme Yin (2013), ideal em situações organizacionais reais em que o pesquisador não tem controle dos fenômenos.

\section{Caracterização da Empresa:}

A empresa em estudo pertence ao ramo alimentício e encontra-se localizada na Região Sul do País. $\mathrm{Na}$ empresa são abatidos e industrializados diariamente cerca de 6000 suínos, que são transformados em produtos embutidos, defumados, congelados e salgados, totalizando mensalmente uma produção aproximada de 10000 toneladas de produtos industrializados.

Processo de Fabricação:

O processo de fabricação da linguiça toscana (Figura 1 - Figura meramente ilustrativa), inicia com a chegada da matéria-prima (cortes de suíno e toucinho) na etapa de pesagem. A pesagem inicial é necessária devido ao preparo da carne ser realizado por batelada. A seguir, os cortes de suíno são moídos e enviados para a etapa de mistura. Na mistura o tempero é adicionado à carne. Na sequência, a carne é levada a uma câmara fria para a etapa de cura. Depois da cura o produto é levado para etapa de embutimento, realizado de forma automática. Nesta etapa acontece o preenchimento das tripas e a formação dos doze gomos. Em seguida, a lingüiça é colocada manualmente na embalagem plástica e embalada a vácuo. Depois a embalagem é pesada para verificar se o peso está dentro dos padrões adotado pela empresa. Finalmente, o produto é enviado para o congelamento.

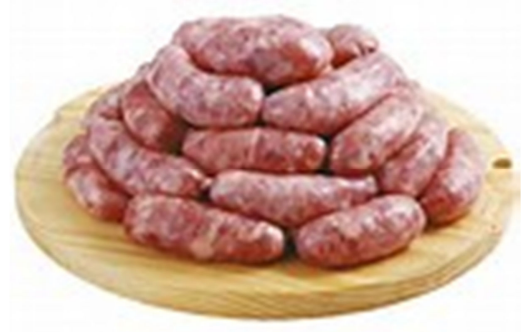

Figura 1: Lingüiça toscana

Fonte: MORESCO (2017). 
O fluxograma do processo pode ser observado na Figura 2.
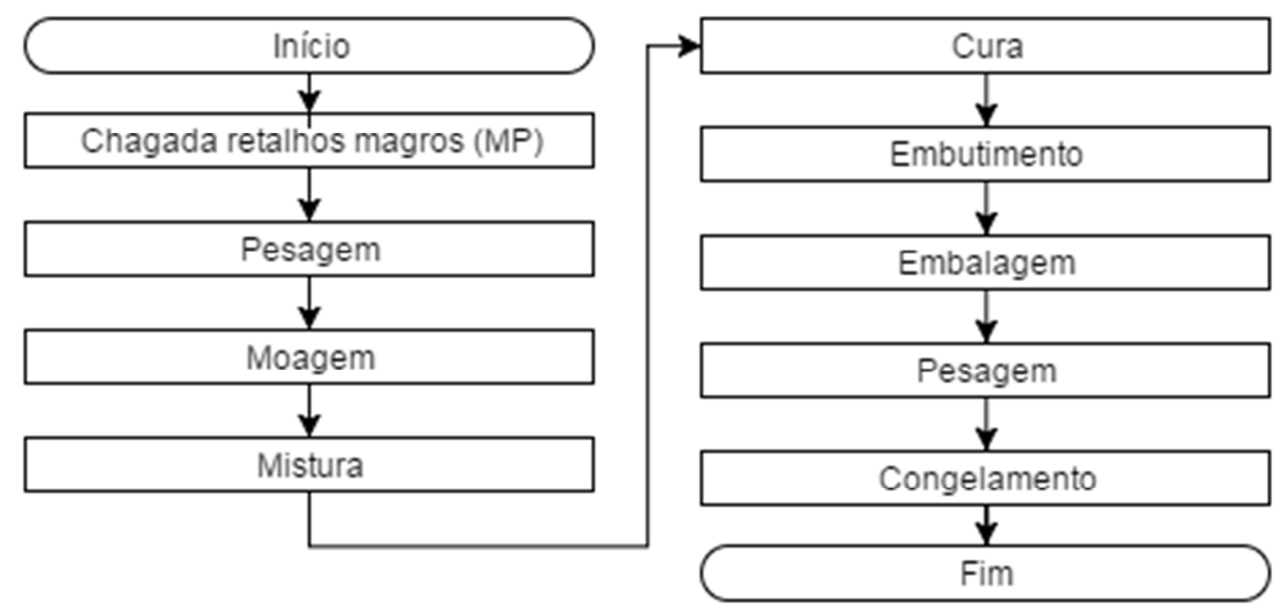

Figura 2: Fluxograma do processo de fabricação de lingüiça toscana (1 kg) Fonte: O Autor.

Gráficos de Controle:

O gráfico de controle consiste na plotagem de amostras ordenadas no tempo, que são entendidas através de linhas horizontais, possuindo uma linha central (LC) ou também denominada linha média (LM), além de outras duas linhas chamadas de limite superior de controle (LSC) e limite inferior de controle (LIC), (Figura 3).

O gráfico ou carta de controle tem como objetivo detectar desvios de parâmetros representativos do processo. Com sua utilização pode-se reduzir os custos de produção e a quantidade de produtos fora de especificações (ALENCAR et al., 2007).

Este gráfico tem por objetivo (RAMOS, 2013):

a) verificar se o processo em análise é estatisticamente estável, sem há presença de causas especiais;

b) verificar se o processo continua estável, indicando quando se deve atuar sobre ele;

c) permitir o aprimoramento constante do processo, por meio da redução de sua variabilidade.

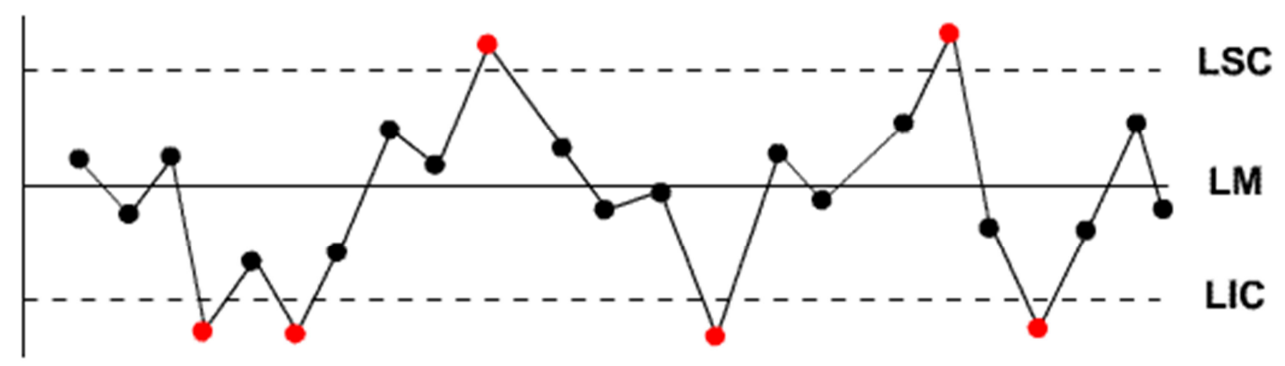

Figura 3: Gráfico de controle

Fonte: O Autor. 
Segundo Montgomery (2001), o processo está fora de controle se tiver:

a) 1 ou mais pontos acima do LSC ou abaixo do LIC;

b) 9 pontos consecutivos na zona $\mathrm{C}$ ou no mesmo lado do LC (Figura 4);

c) 6 pontos consecutivos, todos aumentando ou todos diminuindo;

d) 14 pontos consecutivos alternando para cima e para baixo;

e) 2 de 3 pontos consecutivos na zona A ou além dela;

f) 4 de 5 pontos consecutivos na zona B ou além dela;

g) 15 pontos consecutivos na zona $\mathrm{C}$ (tanto acima quanto abaixo do $\mathrm{LC}$ );

h) 8 pontos consecutivos na zona $\mathrm{B}$.

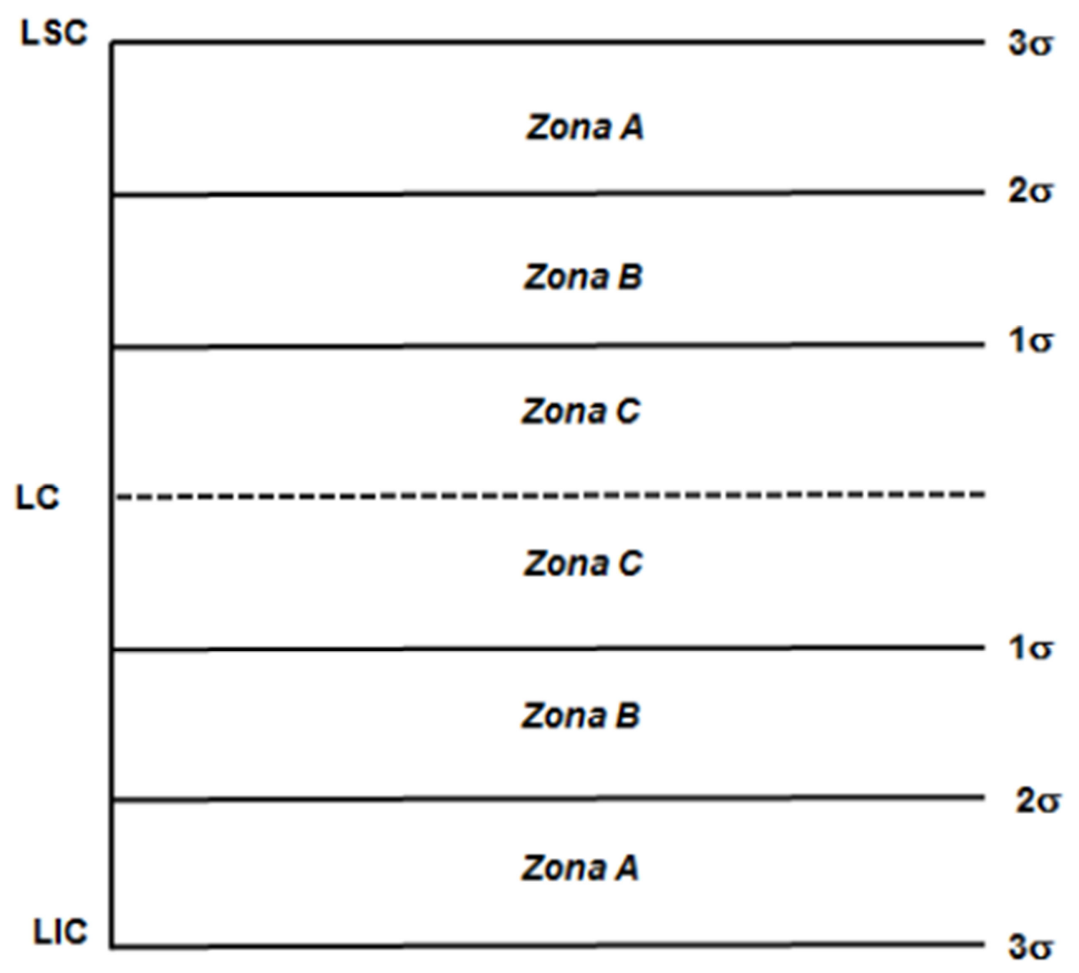

Figura 4: Zonas A, B e C do gráfico de controle. Fonte: O Autor.

Gráfico de Observações Individuais e Amplitude Móvel:

De acordo com Costa et al. (2010), os gráficos de Xi e MR (valores individuais e amplitude móvel), são utilizados em casos em que as amostras são de tamanho $\mathrm{n}=1$. A linha média para o gráfico de $\mathrm{X}$ é a média do processo (valor alvo ou estimativa da média em controle do processo) e os limites de controle distam três vezes o desvio padrão do processo. Geralmente os gráficos de $\mathrm{X}$ e $\mathrm{MR}$ são utilizados em processos automatizados, nos quais ocorre inspeção de $100 \%$ da produção, ou em processos onde a taxa de produção é baixa.

Capacidade de Processo:

A capacidade de processo, ou também expressa por capabilidade de processos, refere-se à como um processo está agindo especificamente, isto é, se ele é capaz de produzir corretamente de acordo com que foi especificado pelo cliente (COSTA et al., 2010). 
Carvalho (2013), mostra que há uma diferença entre controle estatístico e estudo de capacidade, sendo o primeiro proposto à monitorar e compreender ao longo do tempo a estabilidade do processo. Já o estudo de capacidade, objetiva descobrir se o processo está prestando serviço ou produzindo itens conformes, ou seja, de acordo com as determinações feitas pelos clientes.

O índice de capacidade do processo $\left(\mathrm{C}_{\mathrm{pk}}\right)$ é calculado através da Equação 1:

$$
C_{P L}=\frac{(\mu-L T I)}{3 \sigma}, C_{P U}=\frac{(L T S-\mu)}{3 \sigma}, C_{p k}=\operatorname{MIN}\left(C_{P L}, C_{P U}\right)
$$

Onde: LTS: Limite de tolerância superior; LTI: Limite de tolerância inferior; $\mu$ : Média do processo e $\sigma:$ Desvio padrão estimado.

No Quadro 1 apresenta-se a classificação do processo através do índice $\mathrm{C}_{\mathrm{pk}}$.

Quadro 1: Classificação do processo

\begin{tabular}{c|c}
\hline Classificação & Valor de \\
\hline Capaz & $\geq 1,33$ \\
Razoavelmente capaz & $\leq 1,33$ \\
Incapaz & $<1$ \\
\hline
\end{tabular}

Fonte: Adaptado de COSTA et al., (2010).

\section{RESULTADOS E DISCUSSÕES}

Inicialmente, utilizou-se do gráfico de controle individual Xi e da amplitude móvel MR para avaliar se o processo de produção de lingüiça toscana estava sob controle estatístico (Figura 5). Isto é, não apresentava causas especiais.

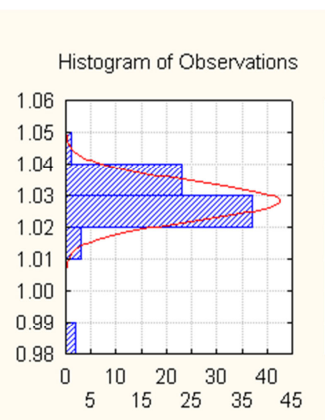

$X$ and Moving $R$ Chart; variable: Peso

$X: 1.0282$ (1.0282); Sigma: .00619 (.00619); n: 1.

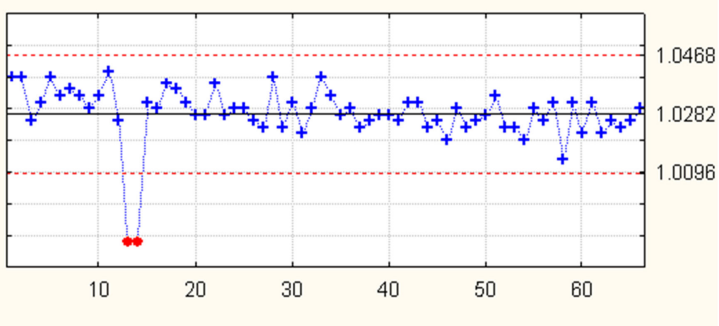

Histogram of Moving Ranges

Moving R: .00698 (.00698); Sigma: .00528 (.00528); n: 1.
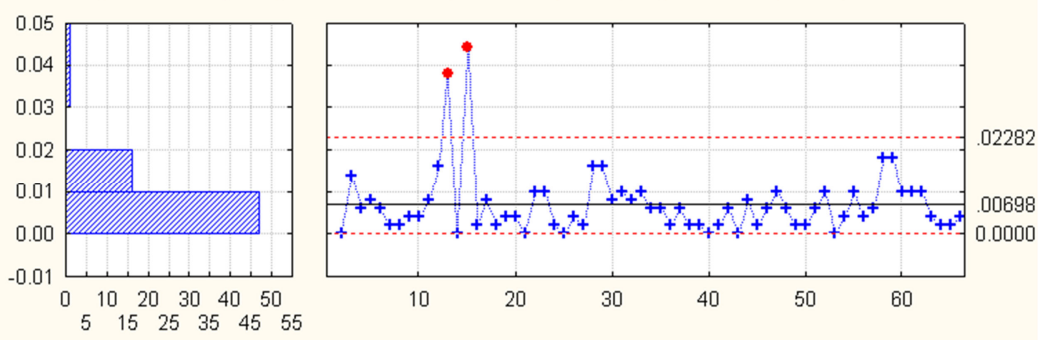

Figura 5: Gráfico inicial de controle individual Xi e da amplitude móvel MR Fonte: O Autor. 
Observa-se, por meio do gráfico $\mathrm{X}$ apresentado na Figura 5, que os dados obtidos do processo apresentavam duas causas especiais, devido a problemas de falta de carne no processo de embutimento automático (SILVA et. al., 2008). Depois de eliminadas as causas especiais do processo, os gráficos foram refeitos (Figura 6) (SILVA et. al., 2008).

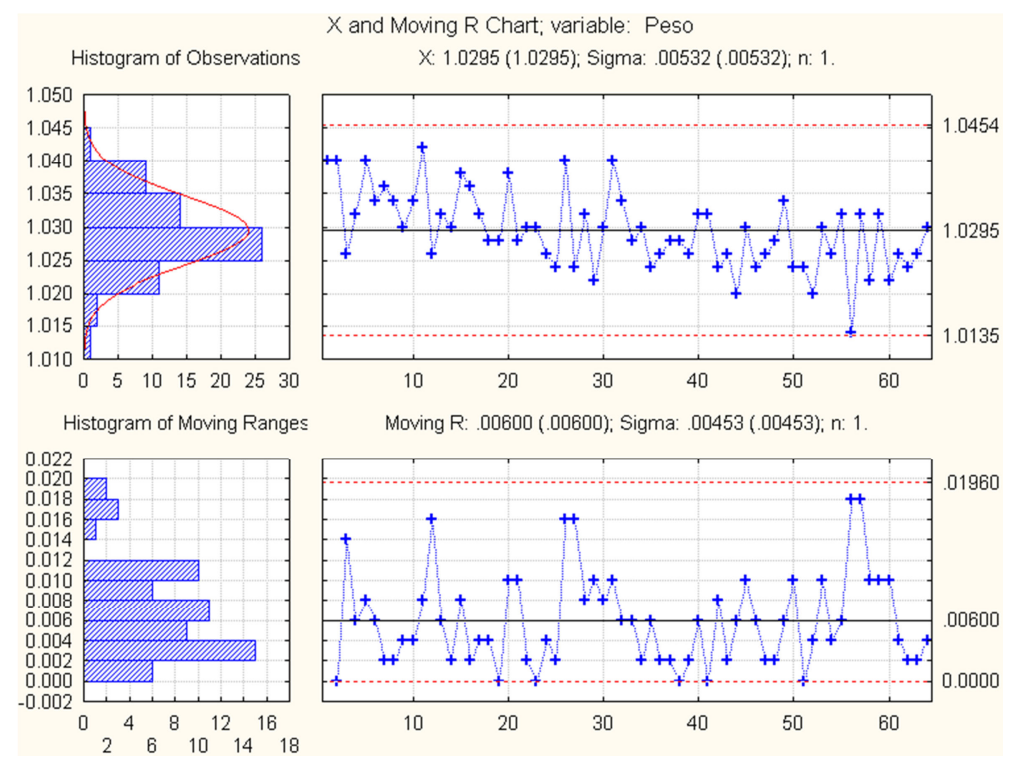

Figura 6: Gráfico final de controle individual Xi e da amplitude móvel MR Fonte: O Autor.

Pode-se observar, por meio dos gráficos apresentados na Figura 6 que todos os pontos situaram-se entre os limites de controle e exibiram comportamento aleatório, indicando que o processo estava sob controle. Quando todos os pontos encontram-se dentro dos limites de controle, outros padrões de nãoaleatoriedade devem ser investigados (Figura 7).

\begin{tabular}{|c|c|c|c|}
\hline \multirow[b]{2}{*}{$\begin{array}{l}\text { Zones A/B/C: } 3.000 / 2.000 / 1.000{ }^{*} \text { Sigma } \\
\text { Tests for special causes (runs rules) }\end{array}$} & \multicolumn{3}{|c|}{$\begin{array}{l}\text { Peso; Runs Tests (TTHia) } \\
\text { X Chart } \\
\text { Center line: } 1.029469 \text { Sigma: } 0.005317\end{array}$} \\
\hline & \begin{tabular}{|c|} 
from \\
sample
\end{tabular} & $\begin{array}{c}\text { to } \\
\text { sample }\end{array}$ & \\
\hline 9 samples on same side of center & $\mathrm{OK}$ & $\mathrm{OK}$ & \\
\hline 6 samples in row in/decreasing & $\mathrm{OK}$ & $\overline{\mathrm{OK}}$ & \\
\hline 14 samples alternating up \& down & 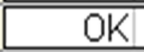 & $\overline{\mathrm{OK}}$ & \\
\hline 2 of 3 samples in Zone $A$ or beyond & $\overline{\mathrm{OKK}}$ & $\overline{\mathrm{OK}}$ & \\
\hline 4 of 5 samples in Zone $B$ or beyond & $\overline{\mathrm{OK}}$ & $\overline{\mathrm{OKK}}$ & \\
\hline 15 samples in Zone $\mathrm{C}$ & 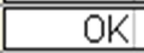 & $\overline{\mathrm{OK}}$ & \\
\hline 8 samples beyond Zone $\mathrm{C}$ & OKK & $\overline{\mathrm{OK}}$ & \\
\hline
\end{tabular}

Figura 7: Testes de padrões de não-aleatoriedade

Fonte: O Autor.

Observa-se que quando trata-se de dados individuais tem que se ter um cuidado especial com a questão da normalidade dos dados. Por meio do teste de Shapiro-Wilk é possível julgar se os dados se ajustam a uma distribuição normal. Observa-se que o $p$-value obtido foi de 0.1341 , maior que 0.05 (Figura 8). Assim, pode-se afirmar com nível de significância de $5 \%$ que a amostra provém de uma população normal. 


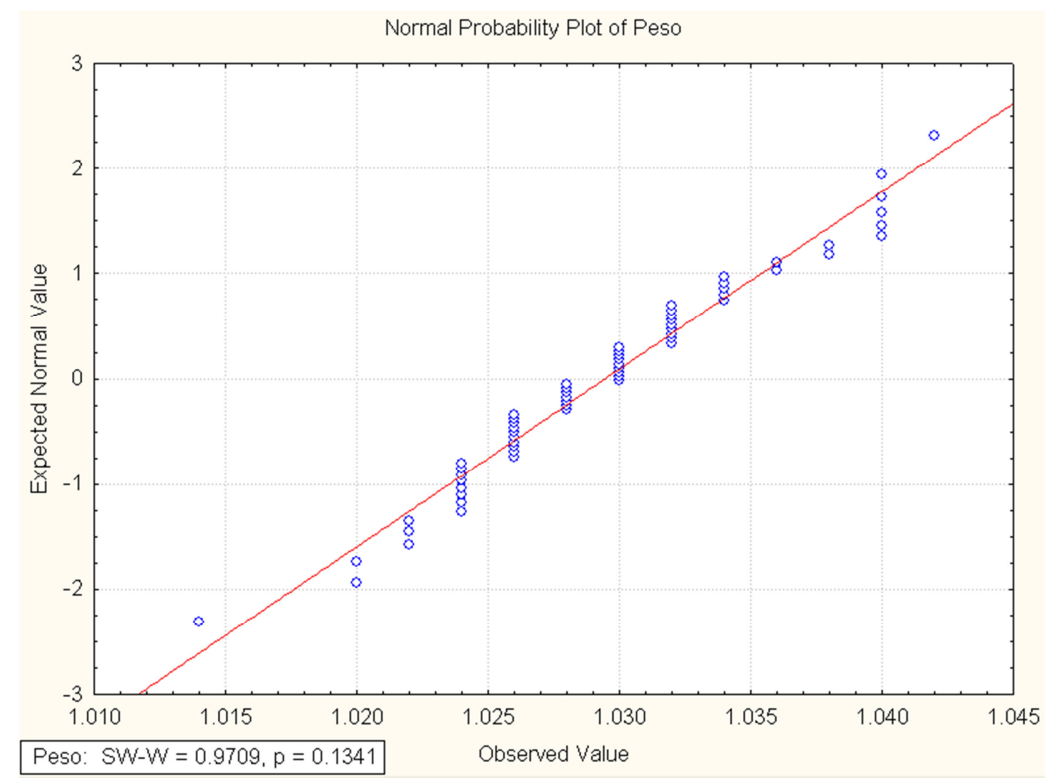

Figura 8: Distribuição dos dados em torno da reta que indica normalidade Fonte: O Autor.

Verificada a estabilidade do processo quantificou-se sua capacidade em atender os limites especificados pela legislação e pela empresa. Segunda a Portaria do Instituto Nacional de Metrologia, Normalização e Qualidade Industrial (INMETRO) no 74 de 1995, os valores admissíveis para a Tolerância Individual $(\mathrm{T})$ da característica da qualidade peso são de 15 gramas, ou seja, o Limite Inferior de Especificação é de 15 gramas. Então, para um produto de $1 \mathrm{~kg}$ o peso mínimo para o consumidor é de 985 gramas. O Limite Superior fica a critério da empresa, neste caso utilizou-se 1050 gramas como limite superior.

Na Figura 9 apresenta-se o histograma e o índice de capacidade $C_{p k}$, para o processo de produção de lingüiça toscana.

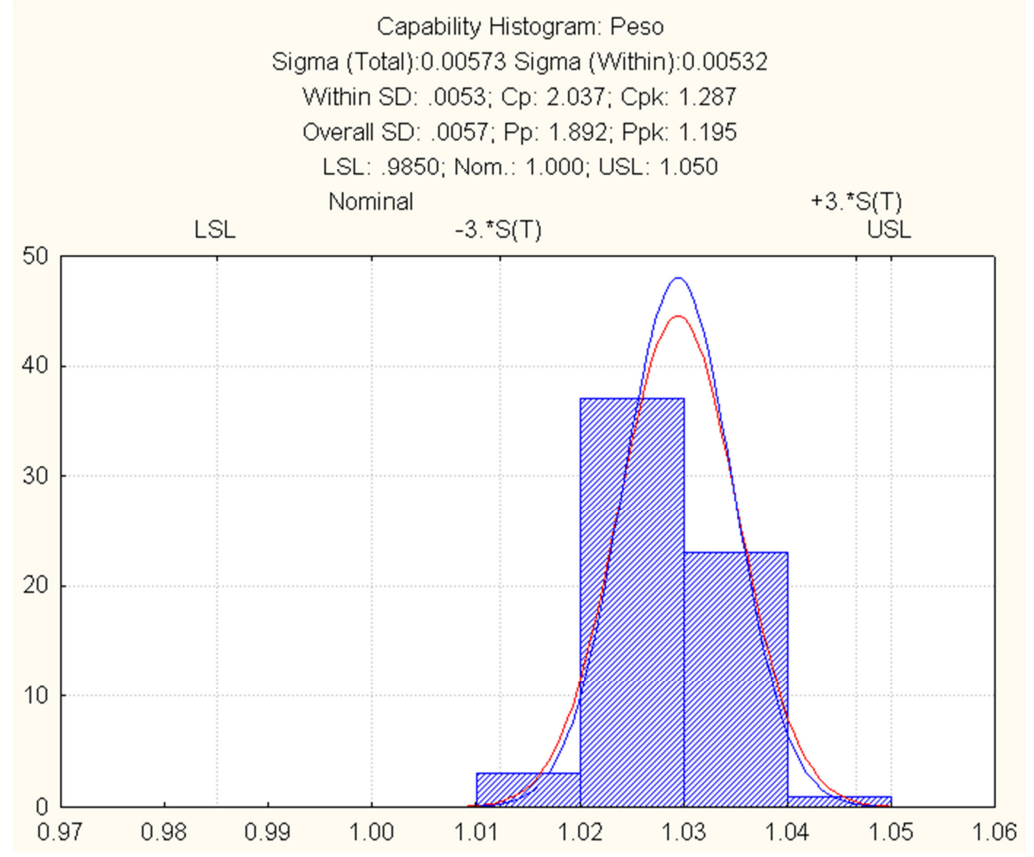

Figura 9: Histograma e Índice de Capacidade $\mathrm{C}_{\mathrm{pk}}$ Fonte: O Autor. 
Observou-se que o índice de capacidade calculado para o processo de produção de lingüiça toscana está acima de um $\left(\mathrm{C}_{\mathrm{pk}}=1,287\right)$, o que classifica o processo como razoavelmente capaz. Cabe se observar que o processo não foi considerado capaz por conta de excesso de produto não pela falta.

\section{CONCLUSÕES}

Neste trabalho apresentou-se as metodologias utilizadas para analisar e verificar as principais causas de variabilidade do peso de embalagens de $1 \mathrm{~kg}$ de lingüiça toscana de uma indústria alimentícia localizada na Região Sul do País.

Observou-se, inicialmente, por meio de gráficos de controle que o processo não estava sobre controle estatístico, apresentando causas especiais. Depois de identificadas e eliminadas as causas especiais do processo os gráficos de controle foram refeitos. Na sequência, observou-se que o índice de capacidade $\mathrm{C}_{\mathrm{pk}}$ está acima de um, classificando o processo como razoavelmente capaz. Concluindo-se que o processo atende as especificações determinadas pela legislação e pela empresa.

A partir desse estudo a empresa, também identificou problemas com a falta de de carne no processo de embutimento automático, o que possibilitou a aplicação de medidas gerenciais corretivas ao processo, aumentando a qualidade do produto final.

\section{REFERÊNCIAS}

ALENCAR, J.; LOPES, C. E.; SOUZA, M. Monitoramento do processo de compressão de comprimidos de captopril utilizando controle estatístico de processo. Rev. Bras. Farm, 2007.

CARVALHO, M. M. Selecionando projetos seis sigma. In: ROTONDARO, R. G. Seis Sigma: Estratégia gerencial para a melhoria de processos, produtos e serviços. São Paulo: Atlas, 2013.

ClubedaCarne Principais tipos de linguiça. Disponível em: < http://www.clubedacarne.com/blog/principais-tipos-de-linguica/> Acesso em: 30 julho de 2017.

COSTA, Antonio Fernando Branco; EPPRECHT, Eugenio Kahn; CARPINETTI, Luiz Cesar Ribeiro. Controle estatístico de qualidade. 2. ed. São Paulo: Atlas, 334 p., 2010.

GIL, A. C. Como elaborar projetos de pesquisa. $4^{\text {a }}$ Ed. São Paulo: Atlas, 2002.

JURAN, Joseph M.. Juran planejando para a qualidade. 2. ed. São Paulo: Pioneira, 1993.

MONTGOMERY, D. C. Introduction to statistical quality control. 4ª Ed. New York: John Wiley, 2001.

MORESCO Linguicinha Toscana. Disponível em: < http://www.jmmoresco.com.br/?content=detalhar\&id=1710> Acesso em: 30 julho de 2017.

OLIVEIRA, T. S.; LIMA, R. H. P. Aplicação do controle estatístico de processo na mensuração da variabilidade em uma usina de etanol. INGEPRO, v. 3, n. 6, 2011.

OLIVEIRA, C. C.; GRANATO, D.; CARUSO, M. S. F.; SAKUMA, A. M. Manual para elaboração de cartas de controle para monitoramento de processos de medição quantitativo em laboratório de ensaios. São Paulo: Instituto Adolfo Lutz, 2013.

PIMENTEL GOMES, F. Curso de estatística experimental. Piracicaba: Degaspari, 2000. 
RAMOS, A. W. Mantendo o processo sob controle. In: ROTONDARO, R. G. Seis Sigma: Estratégia gerencial para a melhoria de processos, produtos e serviços. São Paulo: Atlas, 2013.

SILVA, C. R.; KLIDZIO, R.; ANTONELLO, N. R. B. Aplicação de Controle Estatístico de Processos (CEP) para avaliar o processo de defumação do salame. In.: XXVIII ENEGEP, Rio de Janeiro, 2008

TERRA, N. N. Apontamentos de tecnologia de carnes. São Leopoldo: Editora Unisinos, 218p., 2000.

YIN, R. K. Estudo de caso: planejamento e métodos. Porto Alegre: Bookman, 2013. 